\title{
Detailed mapping of the phosphomannomutase 2 (PMM2) gene and mutation detection enable improved analysis for Scandinavian CDG type I families
}

\author{
Cecilia Bjursell ${ }^{1}$, Jan Wahlström ${ }^{1}$, Kerstin Berg ${ }^{1}$, Helena Stibler ${ }^{2}$, Bengt Kristiansson ${ }^{3}$, \\ Gert Matthijs ${ }^{4}$ and Tommy Martinsson ${ }^{1}$ \\ Departments of ${ }^{1}$ Clinical Genetics and \\ ${ }^{3}$ Pediatrics, Sahlgrenska University Hospital/East, Gothenburg \\ ${ }^{2}$ Department of Neurology, Karolinska Hospital, Stockholm, Sweden \\ ${ }^{4}$ Centre for Human Genetics, University of Leuven, Leuven, Belgium
}

The gene for carbohydrate-deficient glycoprotein syndrome type I (CDG1) has previously been localised by us close to marker D16S406 in chromosome region 16p13.2-3. We also presented data indicating a strong founder mutation associated with a specific haplotype in CDG I patients from western Scandinavia. The phosphomannomutase 2 (PMM2) gene was recently put forward as a likely CDG1 candidate gene. We have now shown that the specific haplotype is associated with the PMM2 mutation 357C $>$ A. Using data from radiation hybrid panel we have refined the position of the PMM2 gene to very close to marker D16S3020 in the interval between D16S406 and AFM282ze1 on the distal side and D16S3087 on the proximal side. Due to the severity of the disease many families request prenatal diagnostic services for CDG I. In the meantime, until the mutation spectrum is fully examined, we propose the combined use of mutation analysis and linkage analysis with polymorphic markers as diagnostic tools for Scandinavian CDG I families requesting prenatal diagnosis. Using this strategy we have to date successfully performed 15 prenatal diagnoses for CDG I.

Keywords: CDG I; PMM2; mutation detection; prenatal diagnosis; sequencing

Correspondence: Dr T Martinsson, Department of Clinical Genetics, Sahlgrenska University Hospital/East, S-41685 Gothenburg, Sweden. Tel: + 4631 374803, Fax: + 4631 842160, E-mail: tommy.martinsson@clingen.gu.se

Received 20 January 1998; revised 12 May 1998; accepted 18 May 1998 


\section{Introduction}

Carbohydrate-deficient glycoprotein syndrome I (CDG I; MIM No. 212065) is a disease characterised clinically by severe involvement of the central and peripheral nervous system. Other organs are also involved, particularly retina, liver, bone, gonads, adipose tissue and skin. Biochemically, CDG type I is characterised by complex defects in the carbohydrate residues in a number of serum glycoproteins. It is an autosomal recessive disorder and the incidence in Sweden is estimated to be $1: 50000 .^{1-4}$ There is a substantial childhood mortality of approximately $15 \%$, and surviving patients develop permanent neurological disability.

The gene for carbohydrate-deficient glycoprotein syndrome, $C D G 1$, was previously localized by us to a $13 \mathrm{cM}$ interval on chromosome $16 \mathrm{p} 13^{5}$ using linkage and recombination analysis in 25 European CDG I families. We could also demonstrate a significant allelic association of CDG1 to microsatellite marker D16S406. Using haplotype data, linkage disequilibrium and geographical distribution of CDG1 alleles from 44 CDG I families, mainly from northern Europe, we could define a $C D G 1$ gene region, less than $1 \mathrm{cM}$ and less than $1 \mathrm{Mb}$ in size. ${ }^{6}$ In that study we found a common haplotype, designated the A-haplotype, which included markers D16S513, AFMa284wd5, D16S768 and D16S406. The haplotype was found in a defined geographical area including the south-western part of Sweden, the southern part of Norway and the eastern part of Denmark, indicating a founder mutation.

Van Schaftingen and Jaeken ${ }^{7}$ found the enzyme phosphomannomutase to be deficient in most cases of CDG1, and recently the gene for phosphomannomutase 2 (PMM2; MIM 601785) was cloned and mapped to the earlier proposed $C D G 1$ gene region. ${ }^{8}$ Most CDG I patients were found to carry mutations in the $P M M 2$ gene and thus the PMM2 was proposed as a strong CDG1 candidate gene.

In this study, we present data on the detailed localisation of the PMM2 gene, the identification of a common PMM2 mutation in Scandinavian CDG I patients carrying the A-haplotype, and data on 15 prenatal diagnoses performed by us using linkage methods, in some causes in combination with the identified PMM2 mutation. The combined use of mutation detection and linkage analysis with markers well localised versus the CDG1 gene will improve prenatal analysis for CDG I families.

\section{Materials and Methods}

\section{CDG I Family Material}

In this study we used DNA from 65 CDG I families. Of these, 37 were from the Scandinavian countries Sweden, Denmark and Norway, and 28 were from non-Scandinavian countries. Forty-four of the families have been presented earlier. ${ }^{5,6}$ The diagnosis of all patients has been based on the clinical and biochemical criteria given by Jaeken et $a l^{1}$ and by Hagberg et $a l{ }^{3}$ When possible (in 10 cases), measurements of phosphomannomutase activity have been performed and found to be low. Of the 65 families, 13 have been analysed for in total 15 prenatal diagnoses using linkage data, in some cases in combination with PMM enzyme data and PMM2 mutation data. Criteria for a family to be subjected to prenatal diagnosis were that the affected child in the family showed the typical clinical and biochemical signs of CDG I. Additional supporting criteria were that the family was showing linkage to the CDG1 gene region or showing the A-haplotype. Also, 120 control chromosomes were tested for the $375 \mathrm{C}>\mathrm{A}$ mutation using DNA from healthy blood donors.

\section{DNA Extraction and PCR Conditions for STRP Analyses}

Genomic DNA was extracted from blood samples anticoagulated with EDTA as well as from chorionic villi material (for prenatal diagnoses) using standard procedures. Short tandem repeat polymorphic markers (STRPs) were analysed with PCR as described earlier ${ }^{5,6}$ using optimal buffer and annealing conditions including incorporation of ${ }^{32} \mathrm{P}-\mathrm{dCTP}$. After PCR, products were separated on a standard sequencing gel ( $6 \%$ polyacrylamide/7 $\mathrm{M}$ urea). The gels were dried and exposed to an X-ray film overnight. The results were scored and haplotypes could be constructed.

\section{Ordering of DNA Markers against a Radiation Hybrid Panel}

Relevant markers earlier mapped ${ }^{9,10}$ to the CDG1 region (D16S418, D16S502, D16S495, D16S513, AFMa284wd5, D16S768, also denoted GATA-p6084, D16S406, AFM282ze1, D16S3020, D16S3087, D16S404, D16S407, D16S414, D16S519, D16S500 and D16S405) were analysed versus the Stanford G3 and the Stanford TNG3 radiation hybrid mapping panels (purchased from Research Genetics Inc, Huntsville, Alabama). In addition the PMM2 gene exon 6 (primer sequences listed below) was included in this study. From each radiation hybrid clone $125 \mathrm{ng}$ DNA was used for PCR, using optimal conditions for each primer pair. The PCR product was separated on a standard $2 \%$ agarose gel with ethidiumbromide, and positive and negative clones could be scored.

\section{PCR and DNA Sequencing of PMM2 Gene}

Sequences, and Restriction Endonuclease Digests of PCR Products

DNA sequencing of the 8 exons in the $P M M 2$ gene were carried out using an ALF DNA Sequencer (Pharmacia, Uppsala, Sweden). All exons were PCR amplified using exon specific primers and exon 5 was amplified in two parts, denoted exon 5:1 and 5:2. The PCR primer sequences used were as described by Matthijs et al. ${ }^{11}$

The forward primers were all flanked by a M13 universal primer (5'-cga cgt tgt aaa acg acg gcc agt-3') and the reverse 
primers were all flanked by a M13 reverse primer (5'-cag gaa aca gct atg ac-3'). In order to avoid misincorporation of dNTPs by the DNA polymerase the Expand High Fidelity PCR system (Boehringer Mannheim, Mannheim, Germany) was used in this amplification reaction. The PCR fragments were purified from dNTPs, enzyme and primers using QIAquick PCR Purification Kit (QIAGEN, Hilden, Germany).

We used approximately $50 \mathrm{ng}$ of the PCR product for the cycle sequencing which was performed using Thermo Sequenase (Amersham, Little Chalfont, Buckinghamshire, England) according to the recommendations of the manufacturer with M13 universal primer (Pharmacia, Uppsala, Sweden).

For rapid detection of the $357 \mathrm{C}>\mathrm{A}$ mutation, exon 5:1 was PCR amplified and the fragments (approximately $200 \mathrm{ng}$ of product) were digested for $3 \mathrm{~h}$ in $65^{\circ} \mathrm{C}$ with $4 \mathrm{U}$ of restriction endonuclease Tru9I (Boehringer Mannheim, Mannheim, Germany). The digested products were separated on a $5 \%$ agarose gel (4\% NuSieve, USB/Amersham, LIFE SCIENCE, Cleveland, Ohio, 1\% Agarose-ultra pure, Gibco BRL/Life Technologies, Paisley, Scotland) and analysed.

\section{Prenatal Diagnoses}

For prenatal diagnoses in CDG I families we used a selection of 15 markers (D16S418, D16S502, D16S495, D16S513, AFMa284wd5, D16S768, D16S406, D16S3020, D16S3087, D16S404, D16S407, D16S414, D16S519, D16S500 and D16S405) located in the CDG1 gene region. Maps of the region have been published. ${ }^{9,10}$ Markers D16S768, D16S406, D16S404 and D16S407 have been used in all prenatal diagnoses, whilst the other markers were used when more information was needed to achieve full information for markers flanking the CDG1 gene. The markers AFMa284wd5, D16S768 and D16S406 were used to detect families carrying the A-haplotype. ${ }^{6}$ Marker D16S404 is the first marker proximal of the A-haplotype, whilst D16S495 and D16S502 are the closest markers on the distal side of the A-haplotype. Recently, analysis of PMM2 mutation 357C > A was also included in the prenatal analyses. These analyses were performed as described above.

\section{Results}

\section{Radiation Hybrid Mapping of STRP Markers and PMM2 Gene Sequences in the CDG1 Gene Region, and Analysis of a Family with a Specific Recombination Event}

Fifteen polymorphic markers in the CDG1 gene region ranging from D16S418 to D16S405 and the PMM2 gene were analysed using the Stanford G3 and the TNG3 radiation hybrid mapping panels. Eleven of the 15 markers were found to be in the core region close to PMM2 and they were mapped relative to PMM2 and to each other in detail (Figure 1a). For the Stanford G3 panel we found 21 of the 83 clones positive for at least one of these markers and the order of the markers could be constructed, based on the pattern of positive clones (Figure 1a). Marker D16S502 was placed distal to D16S495, which differs from our earlier data, but is in better agreement with all our information available at present. Also in two families presented earlier, 13 and $39,{ }^{5,6}$ there were recombinations strongly suggesting that D16S502 locates distal to D16S495. The order of the markers D16S513, AFMa284wd5 and D16S768 could not be determined with the G3 panel, because of the low resolution of the panel for markers situated close to each other. However, the TNG3 panel could resolve them (data not shown, but available on request). Also the fact that four A-haplotypes do not have the allele 5 at position D16S513 (Table 1) strongly suggests that this marker is situated more distally. The data indicate that the order of these three markers is tel-D16S513-AFMa284wd5-D16S768-cen. Also the PMM2 gene and marker D16S3020 could not be resolved on the Stanford G3 panel. The PMM2 gene location thus maps in close vicinity to marker D16S3020, proximal to D16S406 and distal to D16S3087. These three markers are all included in the extended Généthon map. ${ }^{9}$ In addition, a Généthon STRP marker not included in the Généthon map, AFM282ze1, is located between marker D16S406 and $P M M 2$. In conclusion, markers AFM282ze1 and D16S3087 are the closest markers flanking the PMM2 gene on the distal and proximal side, respectively.

On analysing the haplotypes in the CDG1 gene region in the material, one family (18) was found to have a recombination event just distal to the $C D G 1$ gene (Figure 1b). The affected child carries the A-haplotype in one of the alleles. The unaffected child in the family carries the same two haplotypes as the affected child for markers distal to AFM282ze1. However, due to a recombination event in the paternally derived allele, the two children differ in one haplotype from marker AFM282ze1 and markers proximal of it. This is in agreement with a position of the CDG1 gene proximal to marker D16S406. The complete marker order best fitting all our data is presented in Figure 1c. Analysis of patients carrying the previously described A-haplotype showed that all these patients shared the same allele also for STRP markers D16S3020 and D16S3087 (data not shown). The specific A-haplotype therefore ranges from the distal marker cluster D16S513-AFMAa284wd5-D16S768 to the proximal marker D16S3087 which spans $1.7 \mathrm{cM}$ on the Généthon map. ${ }^{9}$ 
A
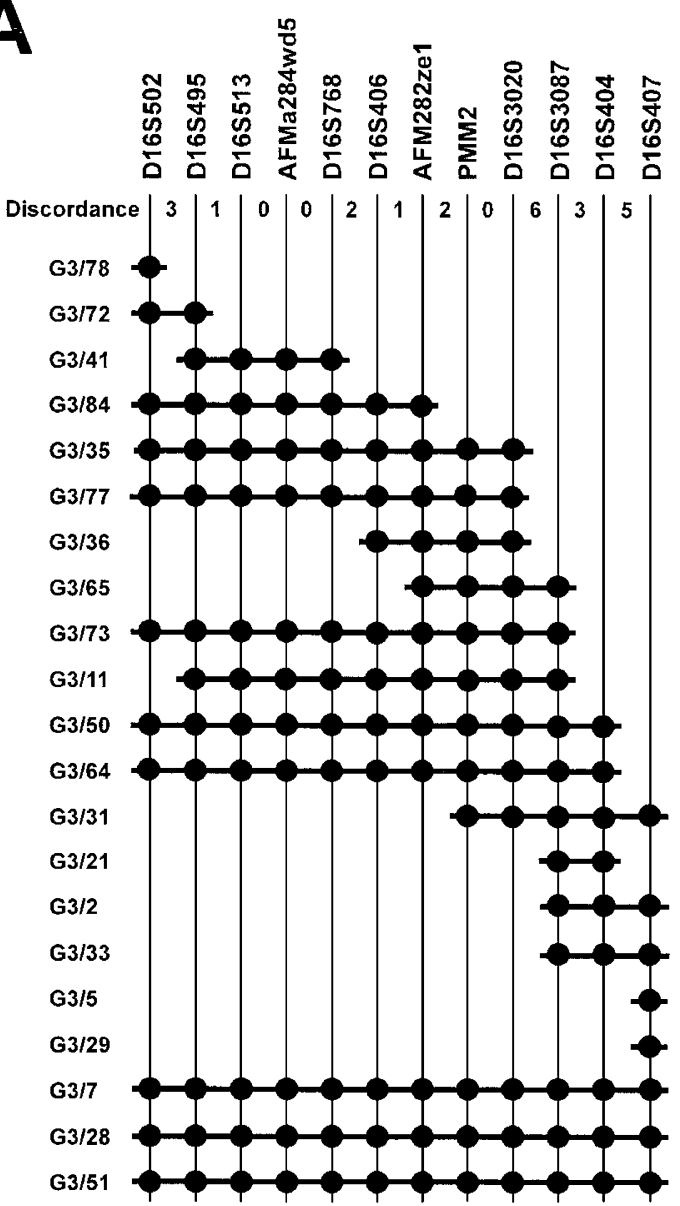

B

D165502

D16 5495

D16S495

AFMa284wd5

D165768

D165406

AFM282ze 1

PMM2 357C $>$ A

D1653020

D1653087

D16S404

D165407

D165519

D16S414

D16S497

D165500
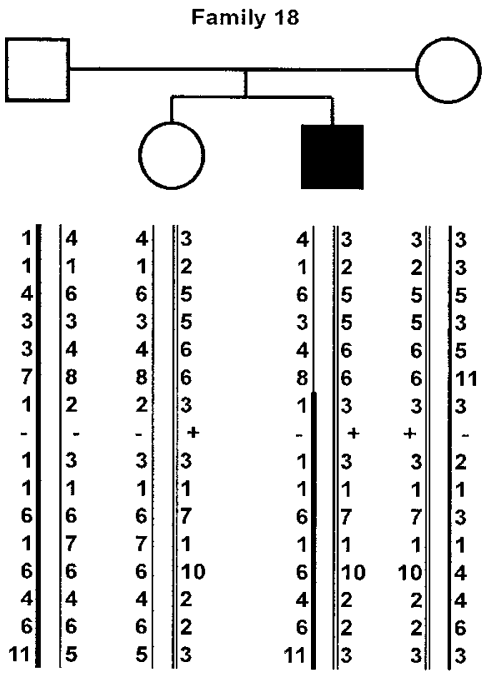

C

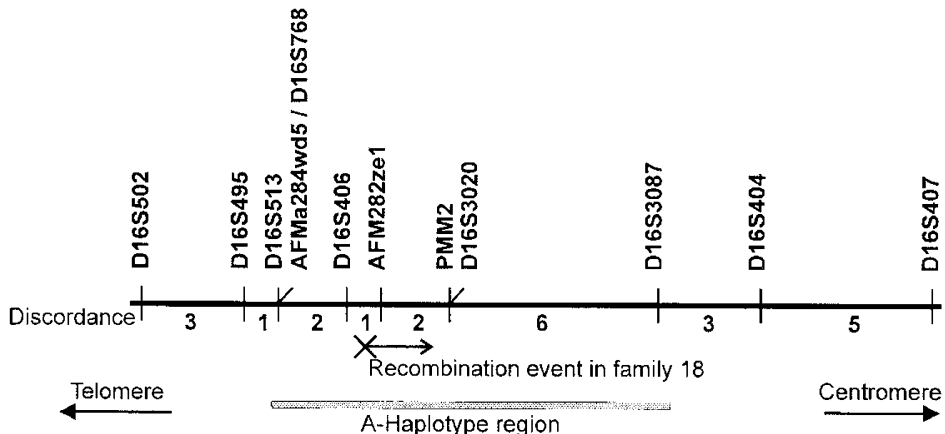

A-Haplotype region

Figure 1 Detailed mapping of the PMM2 gene relative to polymorphic markers in the CDG1 region. a CDG1 gene region markers and PMM2 analysed against the radiation hybrid panel Stanford G3. Filled circles indicate presence of sequences from the marker in the hybrid clone. Only hybrid clones containing at least one of the used markers are included. Figures below markers indicate number of clone discrepancies between the adjacent markers. $\boldsymbol{b}$ Haplotype analysis of a CDG I family with a highly informative recombination in the vicinity of the CDG1 gene. $c$ Combined data of marker positions in the CDG1 gene region. The recombination event in family 18 excluding the region distal of D16S406 as CDG1 region is indicated by an X. The extension of the conserved A-haplotype is indicated at the bottom. Distances as in Figure 1a.

Table 1 Haplotype analysis of CDG1 chromosomes carrying the $357 \mathrm{C}>\mathrm{A}$ mutation in the $P M M 2$ gene

\begin{tabular}{llllllllllllllllllllllllllll}
\hline Family number & 2 & 8 & 13 & 14 & 17 & 17 & 18 & 20 & 22 & 23 & 29 & 41 & 47 & 42 & 8 & 9 & 31 & 4 & 7 & 57 & 33 & 21 & 43 & 53 & 66 \\
Paternal/Maternal & $M$ & $M$ & $P$ & $M$ & $P$ & $M$ & $M$ & $M$ & $P$ & $M$ & $M$ & $P$ & $P$ & $P$ & $P$ & $M$ & $M$ & $P$ & $P$ & $P$ & $P$ & $P$ & $P$ & $n d^{a}$ & $P$ \\
\hline D16S513 & 5 & 5 & 5 & 5 & 5 & 5 & 5 & 5 & 5 & 5 & 5 & 5 & 5 & 5 & 4 & 2 & 4 & 4 & 4 & nd & 3 & 6 & 2 & nd & nd \\
AFMa284wd5 & 5 & 5 & 5 & 5 & 5 & 5 & 5 & 5 & 5 & 5 & 5 & 5 & 5 & 5 & 5 & 5 & 5 & 5 & 3 & nd & 3 & 3 & nd & nd & 3 \\
D16S768 & 6 & 6 & 6 & 6 & 6 & 6 & 6 & 6 & 6 & 6 & 6 & 6 & 6 & 6 & 6 & 6 & 6 & 6 & 5 & nd & 4 & 4 & 3 & nd & 3 & \\
D16S406 & 6 & 6 & 6 & 6 & 6 & 6 & 6 & 6 & 6 & 6 & 6 & 6 & 6 & 6 & 6 & 6 & 6 & 6 & 6 & 6 & 8 & 8 & 4 & nd & 3 \\
PMM2 357C $>$ A & + & + & + & + & + & + & + & + & + & + & + & + & + & + & + & + & + & + & + & + & + & + & + & + & + \\
D16S404 & 3 & 5 & 7 & 7 & 7 & 7 & 7 & 8 & 3 & 6 & 7 & 5 & 8 & 7 & 7 & 7 & 6 & 7 & 6 & nd & 7 & 7 & 3 & nd & 6 \\
\hline
\end{tabular}

and=not determined. In one family (53) it was impossible to deduce the parental origin of the PMM2 357C $>$ A mutation.

bThe shaded area indicates the specific A-haplotype and its correlation to the PMM2 357C $>$ A mutation. 
Sequencing Data of the PMM2 Gene and Rapid Detection of the 357C > A Mutation in Scandinavian CDG I Patients with

\section{A-haplotype}

In a first step, 10 patients, controls and siblings were sequenced using primers flanking all eight exons. This first set of patients was selected to analyse if the A-haplotype was correlated to a specific mutation. We found a base substitution 357C $>$ A (in PMM2, exon 5) described earlier by Matthijs $e t a l^{8}$ inherited together with the A-haplotype in the families (Figure 2 and 3). The $357 \mathrm{C}>\mathrm{A}$ mutation gave a restriction site not present in the normal allele of the sequence $\mathrm{T} \downarrow$ TAA, which can be recognised by the restriction enzyme Tru9I. This enabled a rapid detection method for this specific mutation using PCR amplification of the 5:1 portion of exon 5 followed by Tru9I restriction enzyme digestion and agarose gel separation. The restricted fragments will be for a control individual only the $191 \mathrm{bp}$ band, for the individual heterozygous for the $357 \mathrm{C}>\mathrm{A}$ mutation $191 \mathrm{bp}+167 \mathrm{bp}+24 \mathrm{bp}$, and for a patient homozygous for the mutation the $167 \mathrm{bp}+24 \mathrm{bp}$ bands. In addition there will be a constant fragment of $58 \mathrm{bp}$ (Figure 2a and b; the $24 \mathrm{bp}$ and the 58bp fragments can be difficult to discern on gel).

Two patients in our material were, as described, earlier homozygous for the A-haplotype. ${ }^{6}$ For example, in family 17 the patient is homozygous for the specific A-haplotype, and the parents and the unaffected sibling are all heterozygous for the A-haplotype (Figure 2a). In both this family and the other with a child homozygous for the A-haplotype the patients were in fact found to be homozygous for the 357C > A mutation (Figure 2a, Figure 3). Both parents were heterozygous for the
A

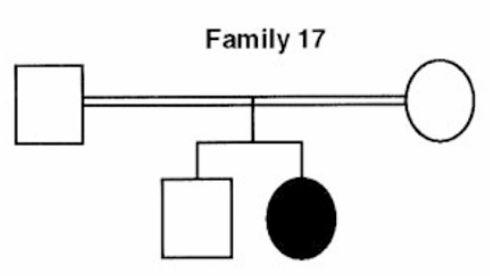

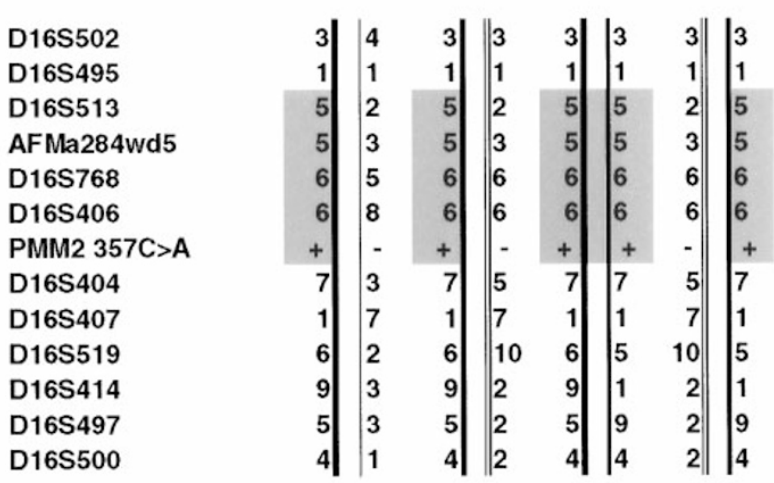

B

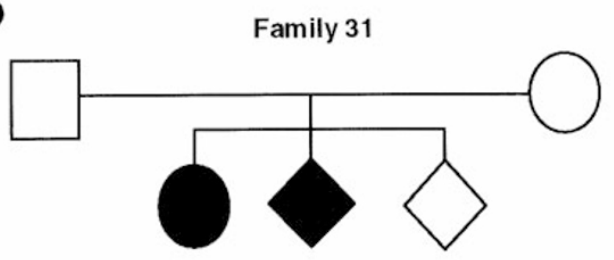

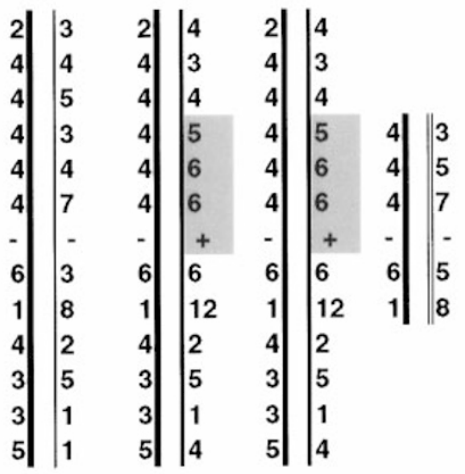

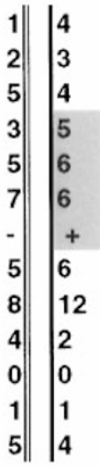
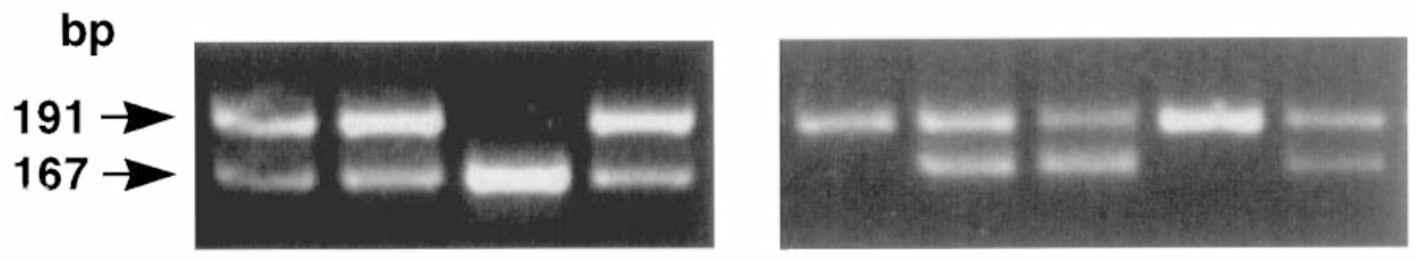

Figure 2 Haplotype and PCR analyses of PMM2 exon 5:1/Tru9I digests in two CDG I families. a Family 17 where the patient is homozygous for the A-haplotype and the $357 C>A$ mutation. $b$ Family 31 with two consecutive prenatal diagnoses, one where the foetus tested as affected and another where the foetus tested as unaffected. 
A

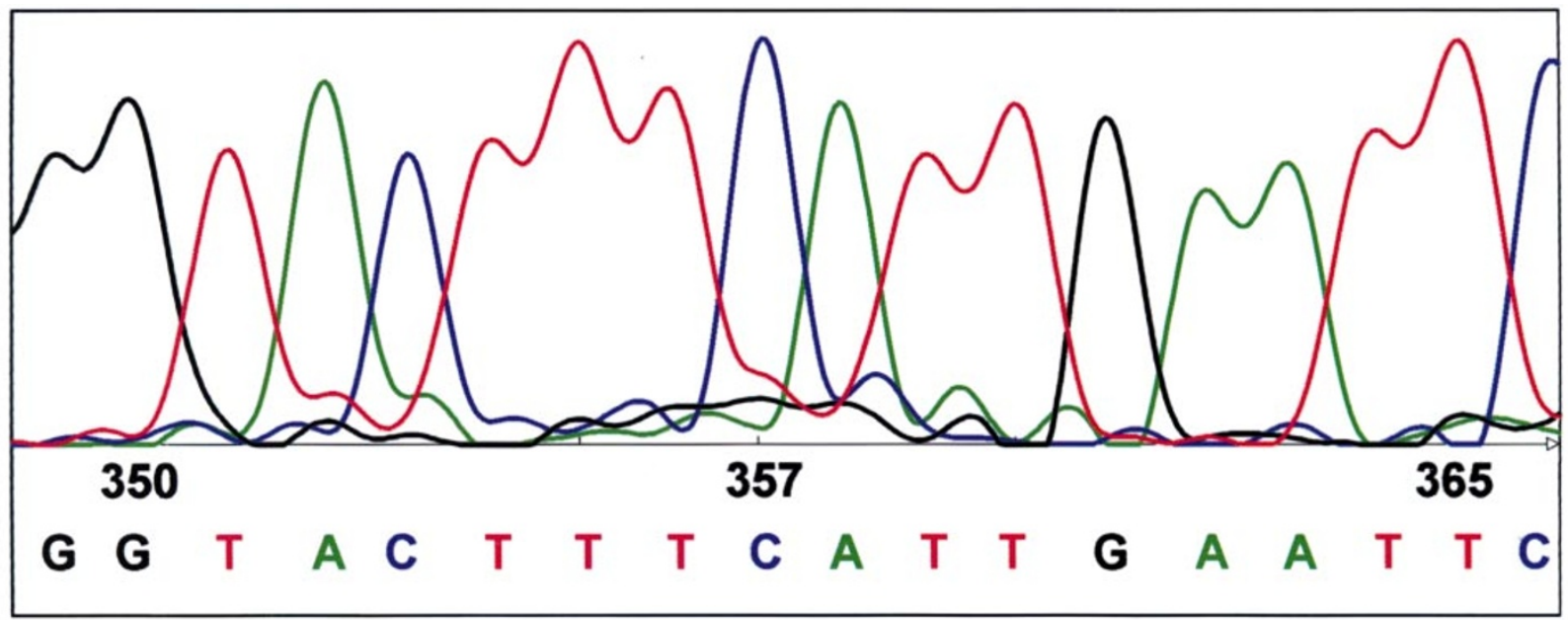

B

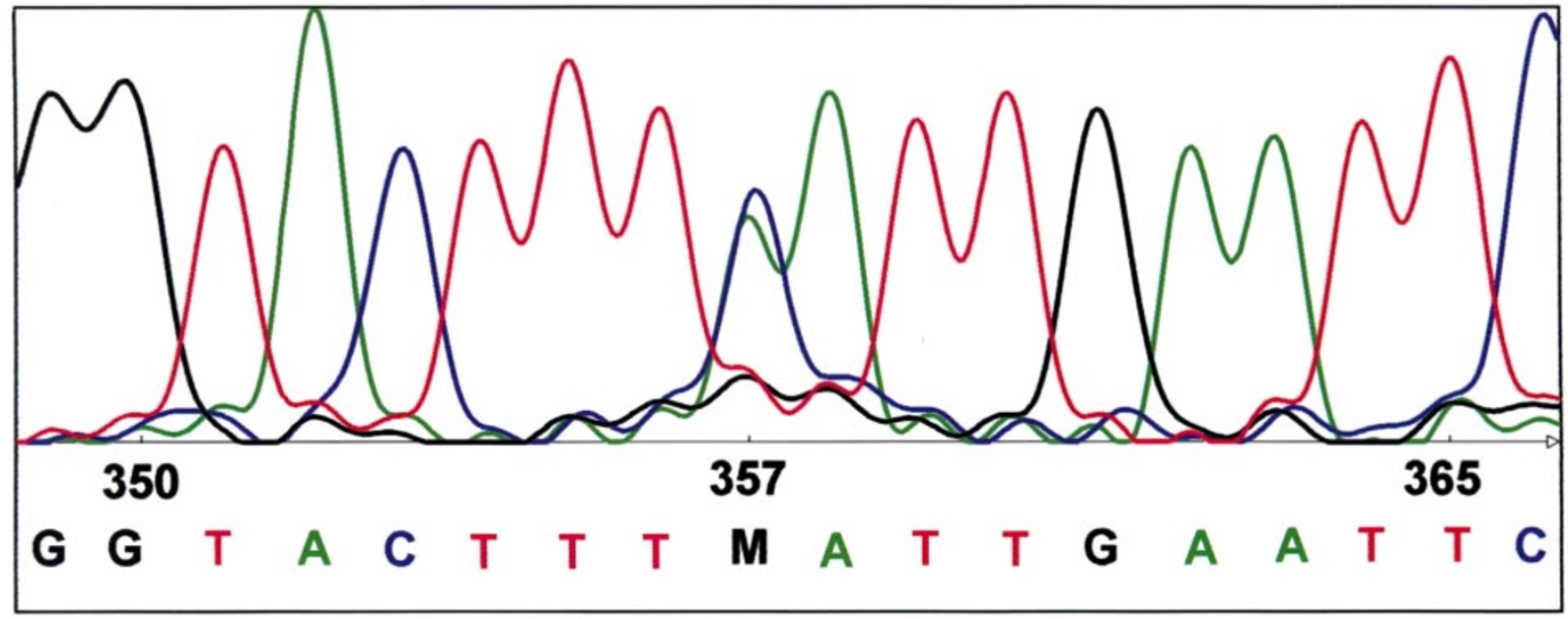

C

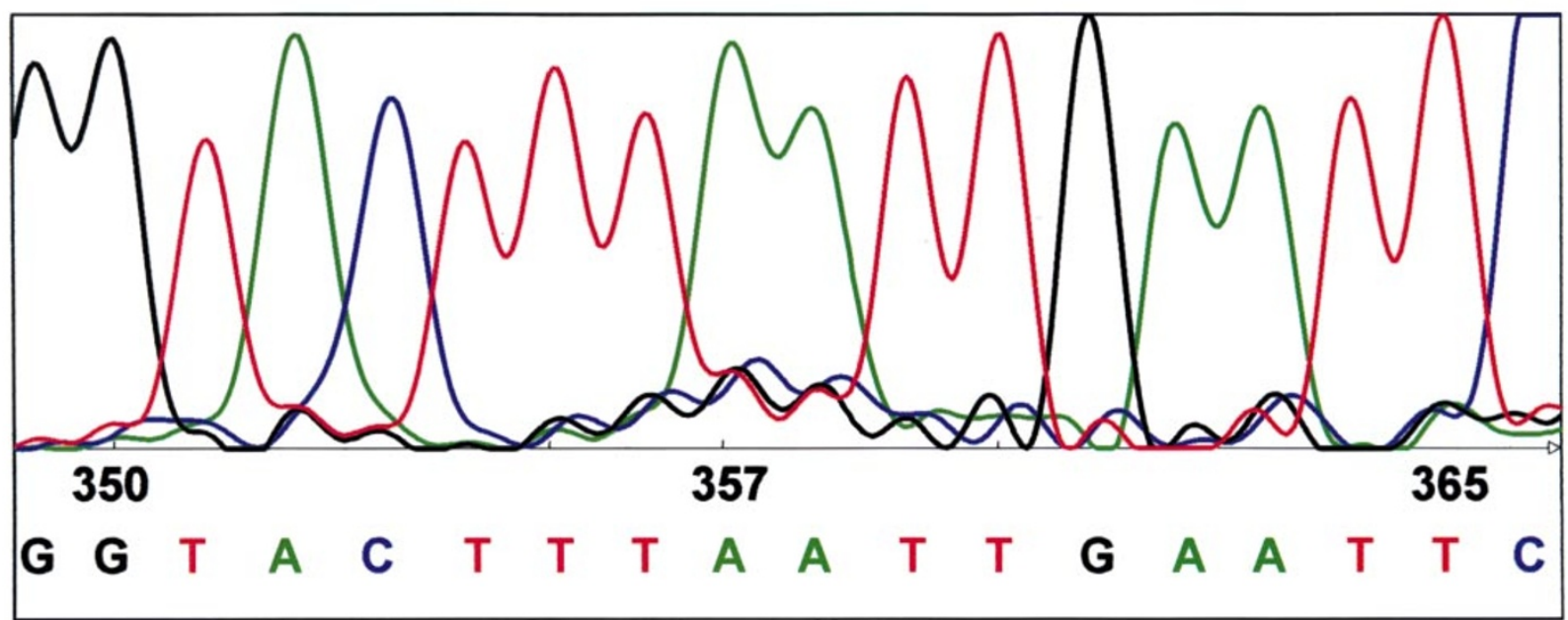

Figure 3 Sequencing data for the PMM2 gene mutation 357C $>$ A (exon 5). a from one healthy control, b one patient heterozygous for the mutation, $c$ one patient homozygous for the $357 C>A$ mutation (patient in family 17). 
357C $>$ A mutation and showed the band pattern of $191 b p+167 b p+24 b p$. The healthy sibling carried the mutation in one allele, whereas the affected sibling had the $357 \mathrm{C}>\mathrm{A}$ mutation in both alleles and displayed the $167 \mathrm{bp}+24 \mathrm{bp}$ band combination (Figure 2a).

All patients in our family material were analysed with the PMM2 exon 5 PCR/Tru9I digestion. In our family material, 18 patients carry the A-haplotype (X-5-6-6; for all but three $\mathrm{X}=5$ ). All A-haplotype alleles carried the $357 \mathrm{C}>\mathrm{A}$ mutation (Table 1 ). All but two are from a defined area from south-western Scandinavia. ${ }^{6}$ Recently we have found one patient from northern Germany and one from England carrying the A-haplotype together with the $357 \mathrm{C}>\mathrm{A}$ mutation. There were also seven alleles carrying the $357 \mathrm{C}>\mathrm{A}$ mutation without the A-haplotype. None of 120 control chromosomes carried the $357 \mathrm{C}>\mathrm{A}$ mutation.

\section{Analysis of 15 Prenatal Diagnoses}

We have performed 15 prenatal diagnoses using STRPs in the $C D G 1$ gene region. Five of the analyses indicated an affected foetus, seven were carriers and three tested as non-carriers. After the cloning of the PMM2 gene some families were also analysed for the $357 \mathrm{C}>\mathrm{A}$ mutation. In all cases where both techniques were used the mutation data were in complete agreement with the linkage data (Figure 2b). All the tested children born six so far - have been confirmed as biochemically and clinically healthy as predicted by the prenatal diagnoses. In one case where the prenatal diagnosis predicted an affected child there was a possibility to analyse material from the interrupted pregnancy for PMM enzyme levels. ${ }^{12}$ The levels were low and thus corroborated the prenatal DNA analysis. Data from one family which had been subjected to CDG I prenatal diagnoses on two occasions are shown in Figure 2. The first pregnancy showed an affected foetus and the pregnancy was interrupted. The second pregnancy, one year later, tested as healthy. The child was recently born and was healthy at birth as predicted.

\section{Discussion}

Due to the severity of CDG I many families with one or more affected children have requested prenatal diagnosis for the disease. Early attempts to perform prenatal diagnosis by analysing patterns of transferrin isoforms in foetal blood were unsuccessful. ${ }^{13,14}$ In contrast, prenatal diagnostic DNA analyses of genetic disorders are ideally performed using direct detection of the disease causing mutations or, in cases when a gene is not known or when the known gene structure is complex, by linkage analysis. Data on the precise location of the disease gene relative to the polymorphic markers used are essential, as information on markers flanking the gene is desired in order to detect possible recombination events that can affect the result.

We earlier localised the CDG1 gene to a $13 \mathrm{cM}$ portion on $16 \mathrm{p}^{5}$ and detected a strong allelic association to marker D16S406 indicating that the gene is situated close to it. In apparent contrast to this, data from Matthijs et $a l^{15}$ indicated that the gene was localised clearly more proximal, ie close to markers D16S414 and D16S497. In a recent paper we provided supporting data for the more distal location in that the $C D G 1$ gene was associated with a particular haplotype (the A-haplotype) in several CDG I families of western Scandinavian origin. ${ }^{6}$ The A-haplotype was again concentrated in the DNA region close to D16S406. Matthijs $e t a l^{8}$ recently cloned the $P M M 2$ gene and found that it mapped between D16S406 and D16S404. Analysis of gene sequences detected mutations in CDG I patients, and the presented data was thus supportive of the PMM2 being in fact the $C D G 1$ gene.

In this paper, using two radiation hybrid panels, and recombination data from families, we have analysed the location of the PMM2 gene in detail relative to a number of DNA markers (Figures 1a and 1b). The best order of markers according to our data is given in Figure 1c.

From the above descriptions two things become clear. Firstly, there is no longer any discrepancy in the location of the $C D G 1$ gene relative to adjacent markers between the two groups. Secondly, there are several good polymorphic markers, close to, and on both sides of the PMM2 gene that can be readily used in prenatal diagnoses of CDG I families if complete mutation information is not available. A good set of markers (ie highly informative and relatively easy to score) that we recommend be used initially is D16S406, AFMa284wd5 and D16S768 on the distal side, D16S3020 very close to the gene and D16S3087 and D16S404 on the proximal side.

The question of heterogeneity was also discussed by

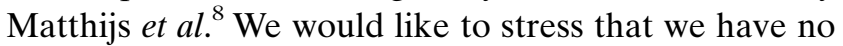
indication of any heterogeneity in the patient material presented here. However, since heterogeneity has been detected in the CDG I patient material studied by others, ${ }^{8,15}$ it is imperative that the index case is ascertained as a PMM2 gene-related patient. This could 
be done preferably by detection of PMM2 mutations in the index case or b linkage or PMM enzyme level measurements.

In analysing PMM2 sequences in patients with $\mathrm{CDG}$ I, we detected one mutation that correlated with the presence of the A-haplotype. This mutation 357C $>\mathrm{A}$ in exon 5 (amino acid change F119L), also described by Matthijs et $a l,{ }^{8}$ was not present in any of 120 normal chromosomes. Since this is one of the most common mutations in our patients (Table 1) we developed a rapid PCR test for this mutation using PCR and Tru9I digestion (Figure 2).

Interestingly, two patients were found to be homozygous for the 357C $>$ A mutation (Figures 2 and 3), both of whom had been shown earlier to be homozygous for the A-haplotype. ${ }^{6}$ Few patients detected so far have proved to be homozygous for a particular mutation, which would have been expected from estimating Hardy-Weinberg equilibrium. ${ }^{11}$ There is therefore no significant deviation from Hardy-Weinberg equilibrium for the $357 \mathrm{C}>\mathrm{A}$ mutation in the population analysed here.

The A-haplotype, including mutation $357 \mathrm{C}>\mathrm{A}$, spans $1.7 \mathrm{cM}$ on the Généthon map $^{9}$ from the distal marker cluster D16S513/AFMAa284wd5/D16S768 to the proximal marker D16S3087 (Figure 1c). As presented earlier we have a concentration of patients in south-western Scandinavia carrying the A-haplotype together with the $357 \mathrm{C}>\mathrm{A}$ mutation. Recently, we identified single A-haplotypes also in one patient from northern Germany and one from England. The extent and spread of the A-haplotype may indicate that this founder mutation is of fairly recent origin. Due to its spreading it has in patient organisations been named the 'Viking-haplotype', since its path is somewhat reminiscent of the routes of ancient Scandinavian seafarers. More data is needed in order to evaluate this.

We have so far provided 15 families with prenatal diagnostic services for CDG I. These were first performed with STRPs only. Recently, data on the PMM2 gene became available ${ }^{8}$ and mutation information has now been included in our analyses when such is available. Due to the high frequency of the $357 \mathrm{C}>\mathrm{A}$ mutation in Scandinavian CDG I patients, and since it is easy to detect with a rapid PCR/Tru9I digest, this analysis has now been included as a first test in CDG I prenatal diagnoses in our area. As we see it, more data is needed until linkage analysis for CDG I patients can be replaced by mutation data only. Several laboratories do not have the capacity to screen the complete coding sequence of a gene for mutations during the limited time that is available for a prenatal diagnostic case. Also, the mutation spectrum and the degree of normal polymorphisms in PMM2 is not yet fully understood. In the paper by Matthijs et $a l^{8}$ it is obvious that most PMM2 mutations are likely to be amino acid substitutions which could be very difficult or even impossible to distinguish from normal polymorphisms. Until more is known about the pathogenesis of CDG I additional data from linkage studies should be pursued.

It now seems clear that the PMM2 gene is in fact the CDG1 gene. Matthijs and co-workers provided strong evidence for it in their recent paper. ${ }^{8}$ In addition, we found the $357 \mathrm{C}>\mathrm{A}$ mutation to correlate strongly with the A-haplotype and also to be present in other CDG I patients in our material. We have mapped in detail the location of the PMM2 gene relative to highly polymorphic STRPs in the region. Using a combination of linkage and mutation analyses we have provided prenatal diagnostic information in 15 cases. In the meantime, until the mutation spectrum is fully understood, we proposed the combined use of mutation analyses and polymorphic markers as diagnostic tools for Scandinavian CDG I families requesting prenatal diagnosis.

\section{Acknowledgements}

We gratefully acknowledge the financial support from the Swedish Medical Research Council (07212, H.S.; 11255, J.W.), the Sven Jerring fund, the SFA, the Karolinska Institute, and the Ronald MacDonald Fund.

\section{References}

1 Jaeken J, Stibler H, Hagberg B (eds): The carbohydratedeficient glycoprotein syndrome. A new inherited multisystemic disease with severe nervous system involvement. Acta Paediatr Scand (suppl.) 1991; 375: 1-71.

2 Jaeken J, Carchon H, Stibler H: The carbohydratedeficient glycoprotein syndromes: pre-Golgi and Golgi disorders?. Glycobiology 1993; 3: 423-428.

3 Hagberg B, Blennow G, Kristiansson B, Stibler H: Carbohydrate-deficient glycoprotein syndromes: Peculiar group of new disorders. Pediatr Neurol 1993; 9: 255-262.

4 Hagberg B, Blennow G, Kristiansson B, Stibler H: Carbohydrate-deficient Glycoprotein Syndromes. In: Vinken P, Bruyn GV (eds). Handbook of Clinical Neurology, Neurodystrophies and Neurolipidoses. Elsevier Science: Amsterdam, 1996, vol 66, pp 623-637. 
5 Martinsson T, Bjursell C, Stibler $\mathrm{H}$ et al: Linkage of a locus for carbohydrate-deficient glycoprotein syndrome I (CDG1) to chromosome 16p, and linkage disequilibrium to microsatellite marker D16S406. Hum Mol Genet 1994; 3: 2037-2042.

6 Bjursell C, Stibler H, Wahlström J et al: Fine mapping of the gene for carbohydrate-deficient glycoprotein syndrome, Type I (CDG1): linkage disequilibrium and founder effect in scandinavian families. Genomics 1997; 39: 247-253.

7 Van Schaftingen E, Jaeken J: Phosphomannomutase deficiency is the cause of carbohydrate-deficient glycoprotein syndrome type I. FEBS Lett 1995; 377: 318-320.

8 Matthijs G, Schollen E, Pardon E et al: Mutations in PMM2, a phosphomannomutase gene on chromosome $16 \mathrm{p} 13$, in carbohydrate-deficient glycoprotein type I syndrome (Jaeken syndrome). Nat Genet 1997; 16: 88-92.

9 Dib C, Faure S, Fizames C et al: A comprehensive genetic map of the human genome based on 5,264 microsatellites. Nature 1996; 380: 152-154.

10 Hudson TJ, Stein LD, Gerety SS et al: An STS-based map of the human genome. Science 1995; 270: 1945-1954.
11 Matthijs G, Schollen E, Van Schaftingen E, Cassiman JJ, Jaeken J: Lack of homozygotes for the most frequent disease allele in carbohydrate-deficient glycoprotein syndrome type IA. Am J Hum Genet 1998; 62: 542-550.

12 Charlwood J, Clayton P, Keir G, Mian N, Young E, Winchester B: Prenatal diagnosis of the carbohydratedeficient glycoprotein syndrome Typ 1A (CDG1A) by a combination of enzymology and genetic linkage after amniocentesis or chorionic villus sampling. Prenat Diagn 1998 in press.

13 Clayton P, Winchester B, di Tomaso E, Young E, Keir G, Rodeck C: Carbohydrate deficient glycoprotein syndrome: normal glycosylation in the fetus. Lancet 1993; 394: 956.

14 Stibler H, Skovby F: Failure to diagnose carbohydratedeficient glycoprotein syndrome prenatally. Pediatr Neurol 1994; 11: 71.

15 Matthijs G, Legius E, Schollen E: Evidence for genetic heterogeneity in the carbohydrate-deficient glycoprotein syndrome type I (CDG1). Genomics 1996; 35: 597-599. 